\title{
Braucht die Ärzteschaft überhaupt eigene Daten?
}

Renato Laffranchi ${ }^{a}$, Urs Stoffel ${ }^{b}$

a General Manager NewIndex

b Präsident des Verwaltungsrats NewIndex, Mitglied des Zentralvorstands der FMH
Immer wieder hört man in Diskussionen die Frage: «Für was braucht die schweizerische Ärzteschaft überhaupt eigene Daten, und wo liegt der Nutzen dieser Datensammlung für die Ärzteschaft?» Oder Antworten wie: «Wir haben doch schon genug Zahlen von den Krankenkassen, dem BAG und dem Bundesamt für Statistik (BFS).» «Eine eigene Datensammlung kostet nur Geld und bringt keinen eigentlichen Mehrwert.»

Im nachfolgenden Artikel zeigen wir auf, warum eigene Daten für die schweizerische Ärzteschaft nötig und wichtig sind, und nicht einfach durch Daten der Versicherer oder des BFS ersetzt werden können.

\section{Wer ist die ärzteeigene Firma NewIndex, und was macht sie?}

Die NewIndex AG wurde im Jahr 2000 im Hinblick auf die künftigen tarifarischen Anforderungen unter TARMED gegründet und ist zu 100\% im Besitz der Kantonalen Ärztegesellschaften, der FMH und der Ärztekasse. Zweck von NewIndex ist die Schaffung eines gesamtschweizerischen Datenpools, unter anderem, um ärzteeigene Daten für die Tarifverhandlungen mit den Versicherern zu haben und um Ärztinnen und Ärzten ein Instrument im Fall von Wirtschaftlichkeitsverfahren zur Verfügung zu stellen. Zu diesem Zwecke wurden mit der Unterstützung der NewIndex gesamtschweizerisch 10 TrustCenter geschaffen. Die Kantonalen Ärztegesellschaften haben mit dem jeweiligen TrustCenter in ihrem Kanton eine vertragliche Abmachung über die Modalitäten der Datensammlung abgeschlossen.
Auswertungen für nationale und kantonale Gesundheitsbehörden, wissenschaftliche Institute und andere im Gesundheitswesen tätige Institutionen.

\section{Datenschutz und Schutz vor Daten- missbrauch haben höchste Priorität}

NewIndex lebt von Daten und damit vom Vertrauen aller Ärztinnen und Ärzte, die freiwillig ihre Daten für die nationale Konsolidierungsstelle NAKO zur Verfügung stellen. Sie erwarten mit Recht, dass NewIndex mit diesen ihr anvertrauten sensiblen Daten sorgfältig, treuhänderisch und datenschutzkonform umgeht. Jede Firma, die dieses «Vertrauenskapital» durch einen unsorgfältigen Umgang mit den Daten aufs Spiel setzt, wird in Kürze tot sein!

Über diesen datenschutzkonformen und sorgfältigen Umgang mit den Daten wacht der «Gatekeeper» der Datenverwendung, das «NAKO-Gremium» und der NAKO-Kodex. Das NAKO Gremium setzt sich aus Vertretern der FMH, der kantonalen Ärztegesellschaften, der verschiedenen Dachverbände, einem Vertreter der TrustCenter, einem Vertreter der NewIndex und einem juristischen Berater zusammen. Der NAKO-Kodex regelt den Zugriff sowie die Nutzung der Daten durch Verbände und Dritte.

\section{Die TrustCenter als Nukleus der nationalen Datensammlung der schweizerischen Ärzteschaft}

Die 10 TrustCenter sammeln dezentral die Abrechnungsdaten der Ärztinnen und Ärzte. Diese Abrechnungsdaten werden dann im nationalen Datenpool
Korrespondenz: Dr. Renato Laffranchi NewIndex AG Leberngasse 19 CH-4600 Olten

\section{NewIndex: Schaffung eines gesamtschweizerischen, ärzteeigenen Datenpools für Tarifverhandlungen mit Versicherern und Instrument bei Wirtschaftlichkeitsverfahren.}

NewIndex unterstützt mit Auswertungen, Analysen und Modellen die beiden nationalen Standesorganisationen KKA und FMH sowohl bei Taxpunktwert(TPW)-Verhandlungen wie auch bei der Revision der TARMED-Tarifstruktur. Daneben übernimmt die NewIndex aber auch «Taylor Made» Datenanalysen, Auswertungen und Datenaufbereitungen für einzelne Kantonale Ärztegesellschaften, einzelne Fachgesellschaften oder Ärztegruppierungen für die verschiedensten Anliegen im Zusammenhang mit ärztlichen Daten; aber auch Analysen und konsolidiert. Die TrustCenter (TC) erfüllen aber noch viel weitergehende Aufgaben. Durch ihre Kundennähe und ihre Kundenbindung schaffen sie Vertrauen, beraten und betreuen die angeschlossenen Ärztinnen und Ärzte, wenn es um ökonomische Aspekte der Praxisführung geht. Nicht wenige Ärztinnen und Ärzte konnten dank den TC ungerechtfertigte Rückforderungen (WZW-Verfahren) der Krankenkassen abwenden. Die TC erfüllen also auch eine wichtige Schutzfunktion für den einzelnen Arzt oder die einzelne Ärztin. 


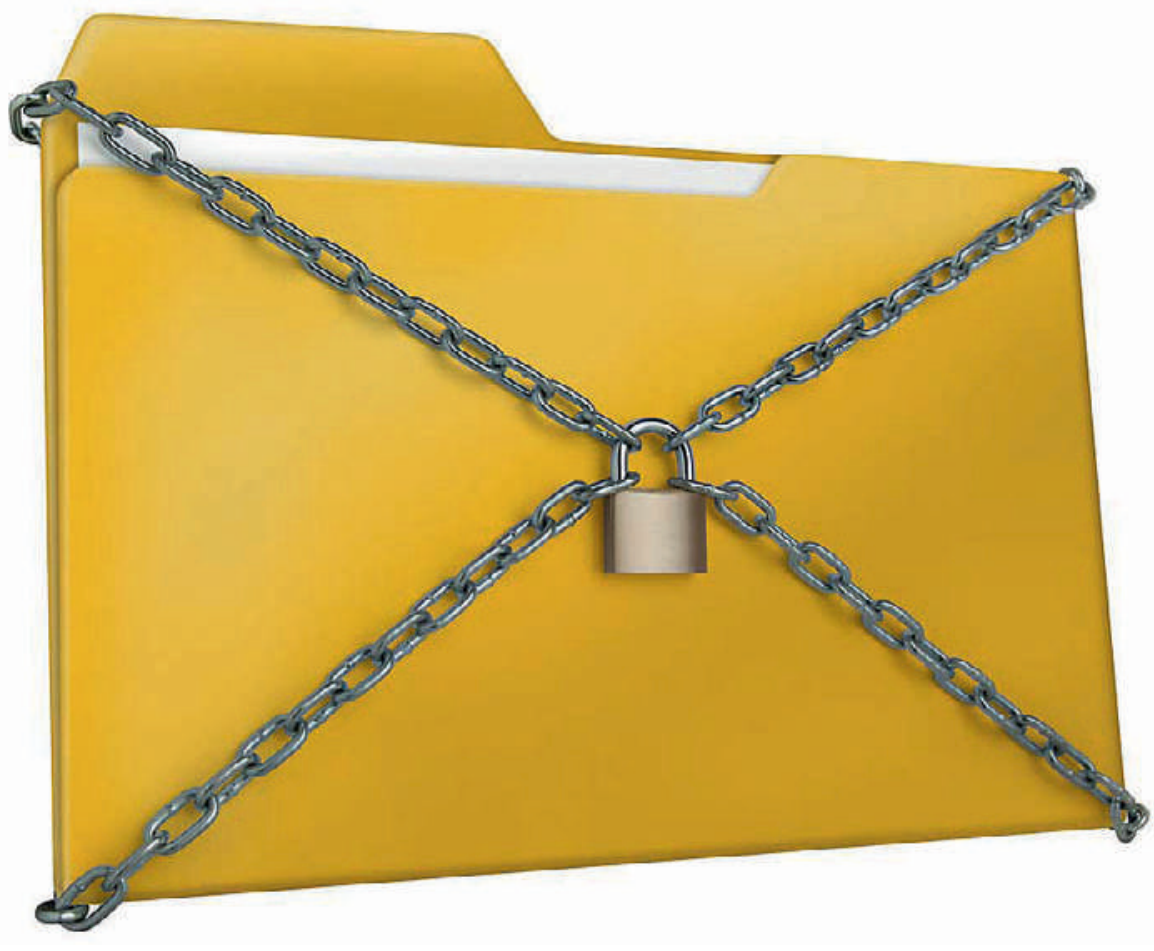

Oberste Priorität bei Newlndex: datenschutzkonformer und sorgfältiger Umgang mit den Daten der Ärzteschaft. vorschläge. Neu in 2013 war NewIndex auch an den Taxpunktwert-Verhandlungen bei der zweiten Versicherereinkaufsgruppe HSK beteiligt.

\section{Newindex als «Backbone» der TARMED Tarifrevision (TARVISION)}

Für die FMH ist NewIndex mit Auswertungen und Analysen in verschiedenen Bereichen tätig. Für das Projekt TARVISION ist eine gute Abdeckung pro Fachspezialität unabdingbar für einen positiven Ausgang des Projektes. Insbesondere können die Anliegen der verschiedenen Fachgesellschaften nur durchgesetzt werden, wenn sie datengestützt plausibilisiert werden.

\section{Newlndex ist das Referenzzentrum für Abrechnungsdaten der Ärzteschaft, die vielseitig für die wichtigen Anliegen der Ärzteschaft eingesetzt werden können}

Anhand von NewIndex-Auswertungen konnten Modelle für neue Labortarife entwickelt werden. Für die Verhandlungen einer margenunabhängigen Abgeltung für die Medikamentenabgabe mit den Versicherern konnte gezeigt werden, dass Konsultationen mit oder ohne Medikamentenabgabe gleich lang dauern.

Aber auch Kantonale Ärztegesellschaften und Fachgesellschaften werden bei Bedarf von NewIndex unterstützt. Im Jahr 2012 konnte NewIndex beispielsweise für die KÄG Schwyz ein umfangreiches Argumentarium entwickeln, mit dessen Hilfe eine Taxpunktwerterhöhung von 2 Rappen erreicht werden konnte. Damals standen wegen einer Datenblockade bei den Versicherern nur ärzteeigene Daten aus der NAKO zur Verfügung, um die Forderungen einer Taxpunktwert-Erhöhung zu begründen.

Ebenso konnte NewIndex für den Kanton Aargau Auswertungen machen und Modelle entwickeln, die schliesslich über eine TPW-Festsetzung des Kantons zu einer Taxpunktwerterhöhung geführt haben. Zudem unterstützt NewIndex den Kanton Aargau im Abstimmungskampf der Initiative zur «Wahlfreiheit beim Medikamentenbezug».

In Zusammenarbeit mit der Fachgesellschaft für medizinische Onkologie konnte das TherapiedichteKonzept erarbeitet werden. Dabei geht es darum, zu zeigen, dass nicht alle Onkologen im gleichen Ausmass onkologische Patienten behandeln, sondern auch zu unterschiedlichen Anteilen Innere Medizin betreiben.

\section{Datengrundlage für eine zukunftsweisende Versorgungsforschung}

Mit Hilfe von OBELISC (Objective Evaluation and Leadership In Scientific Health Data Collection) stösst NewIndex jetzt immer mehr auch in den Bereich der Versorgungsforschung vor: So führte NewIndex in Zusammenarbeit mit dem TrustCenter medkey ein Projekt für die Gesundheitsdirektion des

Sie tragen aber auch in hohem Masse Verantwor-
ung dafür, dass die nationale Datensammlung gewährleistet wird. Die Pflichten und Rechte der TrustCenter werden in Mandatsverträgen mit den Kanto-

\section{Nachfolgemodell erstellt NewIndex eigene Modell- \\ den Versichererdaten gegenübergestellt und vergli- chen. Sollten die Daten nicht die gleiche Entwick- lung zeigen, werden mögliche Erklärungen gesucht
und die Differenzen aufgezeigt. Für das LeiKoV- der Konferenz der \\ Für die KKA übernimmt die NewIndex das gesamte NewIndex die von den Versicherern gelieferten ten auf ihre Stimmigkeit und weist auf allfällige Un- regelmässigkeiten hin. Ergänzend dazu werden die ärzteeigenen Daten aus dem nationalen Datenpool \\ Newlndex als technisches Backoffice Kantonalen Ärztegesellschaften (KKA) technische Backoffice. Im Rahmen der LeiKoV-Ver- handlungen mit tarifsuisse analysiert und überprüft}

Unabdingbare Voraussetzung für die nationale Datensammlung NAKO ist, dass alle TrustCenter so dass die Daten in der NAKO konsolidiert werden konnen und eine Rückwärts- und Seitwärtskompatidurch die TrustCenter auch die jeweils notwendige lange das nicht durch die kantonalen Ärztegesellschaften ermöglicht wird. 
Kantons Schwyz durch. Dabei wurde die Versorgungssituation im Kanton analysiert und dargestellt. Dank OBELISC Daten, einem speziell von der NewIndex geschaffenen Datensubkollektiv, können heute auch externe universitäre Forschungsprojekte unterstützt werden.

Damit wird NewIndex auch zum «Think Tank», der versucht, Fragestellungen zur Zukunft der Gesundheitsversorgung unserer Gesellschaft zu beantworten und $\mathrm{zu}$ antizipieren. Eine echte und $\mathrm{zu}$ kunftsweisende Versorgungsforschung braucht auch das geeignete Datenkollektiv, um valide Aussagen zu machen.

\section{Die standespolitische Bedeutung des nationalen Datenpools}

Wenn die schweizerische Ärzteschaft die Zukunft der Gesundheitsversorgung und des Gesundheitssystems wegweisend und entscheidend beeinflussen will, dann brauchen wir eigene Daten, Auswertungen und Analysen zur Beantwortung der offenen Fragen und vor allem tragfähige Lösungsvorschläge zur zukünftigen Gesundheitsversorgung. Wer anders soll denn diese Zukunft prägen und mitgestalten, wenn nicht die Ärzteschaft? Mit dem nationalen Datenpool der Ärzteschaft haben wir in enger Zusammenarbeit mit den TrustCentern dieses Instrument geschaffen.

\section{Mitgestalten bedeutet auch Verantwortung übernehmen}

Eine Datenbank ist nur so gut wie die Daten, die in diesen Datenpool eingespeist werden. Dies erfordert möglichst viele qualitativ gute Daten, um repräsentative und aussagekräftige Analysen durchführen zu können.
Um dieses Ziel zu erreichen, braucht es gemeinsam akzeptierte Spielregeln und eine solidarische Verteilung der Rechte und Pflichten. Gerade wenn Daten dezentral über verschiedene «Datensammelstellen» (TC) gesammelt werden, muss dies nach einheitlichen Standards und gemeinsam akzeptierten Qualitätsnormen geschehen. Es kann nicht sein, dass jeder eigene Regeln und Normen aufstellt und die gesammelten Daten dann nicht mehr in die gemeinsame Datenbank eingespeist werden können, respektive ihre Vergleichbarkeit verlieren.

\section{Fazit und Blick in die Zukunft}

Die NewIndex hat es sich zum Ziel gesetzt, im Auftrag und zugunsten der Ärzteschaft den mächtigen Datenpool zu nutzen, um den zukünftigen Herausforderungen im schweizerischen Gesundheitswesen mit umsetzbaren Lösungsvorschlägen zu begegnen.

Zum Schluss geht ein grosser Dank an all unsere Mitglieder, die seit Jahren durch ihre Bereitschaft, ihre Daten zur Verfügung zu stellen, die Datenbank überhaupt erst ermöglichen.
Interaktiver Artikel

Wollen Sie diesen Artikel kommentieren? Nutzen Sie dafür die Kommentarfunktion in der OnlineVersion oder sehen Sie nach, was Ihre Kolleginnen und Kollegen bereits geschrieben haben: www.saez.ch/aktuelle-ausgabe/interaktive-beitraege/ 